\title{
Using $\left[{ }^{18} \mathrm{~F}\right] \mathrm{FBAU}$ for imaging brain tumor progression in an F98/tk-luc glioma-bearing rat model
}

\author{
YI-CHUN CHIEN ${ }^{1,2}$, JOHN CHUN-HAO CHEN ${ }^{1,3}$, WEI-CHAN LIN ${ }^{4}$, HUEISCH-JY DING ${ }^{2}$, \\ HSIN-ELL WANG ${ }^{1}$, CHIH-HAO K. KAO ${ }^{5,6}$ and JENG-JONG HWANG ${ }^{1}$
}

\author{
${ }^{1}$ Department of Biomedical Imaging and Radiological Sciences, National Yang-Ming University, Bei-tou 11221, Taipei; \\ ${ }^{2}$ Department of Medical Imaging and Radiological Sciences, I-Shou University, Jiaosu Village, Kaohsiung 82445; \\ ${ }^{3}$ Department of Radiation Oncology, Mackay Memorial Hospital, Tamsui, New Taipei City; ${ }^{4}$ Department of Radiology, \\ Cathay General Hospital, Taipei 10630; ${ }^{5}$ Department of Radiopharmaceutical Production, Buddhist Tzu Chi General Hospital, \\ Hualien 97002; ${ }^{6}$ Department of Radiological Technology, Tzu Chi College of Technology, Hualien 97002, Taiwan, R.O.C.
}

Received March 8, 2014; Accepted March 26, 2014

DOI: 10.3892/or.2014.3256

\begin{abstract}
Deoxy-2- $\left[{ }^{18} \mathrm{~F}\right]$ fluoro- $\beta$-D-arabinofuranosyl)-5bromouracil $\left(\left[{ }^{18} \mathrm{~F}\right] \mathrm{FBAU}\right)$, a substitute for thymine, has been reported as an effective reporter probe by which to trace cellular metabolism with its positron emission. In the present study, a rat xenograft model bearing F98 glioma transfected with dual reporter genes, herpes simplex virus type 1 thymidine kinase (HSV1-tk) and firefly luciferase (luc) was used for monitoring tumor progression by multimodalities of molecular imaging using $\left[{ }^{18} \mathrm{~F}\right] \mathrm{FBAU}$ and D-luciferase as probes. Rat F98 glioma cells were transfected with the pC1-tk-IRES-luc vectors. The selected stable clone was renamed as the F98/tk-luc cell line. Fischer 344 male rats bearing orthotropic F98/tk-luc gliomas in the left brain were used. On day 13 post tumor inoculation, biodistribution, positron emission tomography (PET), magnetic resonance imaging (MRI) and ex vivo autoradiography were performed. The surviving fraction of F98/tk-luc cells treated with $15 \mu \mathrm{M}$ ganciclovir (GCV) was $15.9 \%$, and the uptake of $\left[{ }^{131} \mathrm{I}\right] \mathrm{FIAU}$ in these cells was significantly enhanced when compared with F98 cells. The correlation coefficient of tumor volume vs. the bioluminescence in the F98/tk-luc gliomabearing rats was 0.90 . The biodistribution showed that the accumulation ratios of $\left[{ }^{18} \mathrm{~F}\right] \mathrm{FBAU}$ for glioma-to-normal brain
\end{abstract}

Correspondence to: Professor Jeng-Jong Hwang, Department of Biomedical Imaging and Radiological Sciences, National Yang-Ming University, 155, Sec. 2, Li-Nong Street, Bei-tou 11221, Taipei, Taiwan, R.O.C.

E-mail: jjhwang@ym.edu.tw

Dr Chih-Hao K. Kao, Department of Radiopharmaceutical Production, Buddhist Tzu Chi General Hospital, No 707, Sec. 3, Zhongyang Road, Hualien City, Hualien County 97002, Taiwan, R.O.C.

E-mail:kevin_kao@tzuchi.com.tw

Key words: $\left[{ }^{18} \mathrm{~F}\right] \mathrm{FBAU}, \mathrm{F} 98 / t k$-luc, bioluminescence, MRI, biodistribution, ex vivo autoradiography were $9.16,14.24,5.7$ and 13.7 at $30,60,90$ and 120 min post i.v. injection, respectively. Consistent tumor enhancement of $\left[{ }^{18} \mathrm{~F}\right] \mathrm{FBAU} / \mathrm{PET}$ imaging was also noted from 30-90 min post injection. Ex vivo autoradiography also confirmed significant $\left[{ }^{18} \mathrm{~F}\right] \mathrm{FBAU}$ uptake in tumors. In conclusion, $\left[{ }^{18} \mathrm{~F}\right] \mathrm{FBAU}$ may be used as a PET probe for monitoring glioma progression in animal models and may have potential for clinical use as well.

\section{Introduction}

Glioblastoma multiforme (GBM) is the most aggressive malignant brain tumor in humans with a survival time often less than 1 year even following treatment (1-3). There remains a need for animal models that can be used to assess the efficacy of new and innovative treatments for experimental neurooncology (4,5). The blood-brain barrier (BBB) composed of tight junctions prevents the central nervous system (CNS) from being infiltrated by neurotoxic substances or pharmaceuticals from the peripheral circulatory system. Although the BBB of a tumor-bearing animal is severely compromised (6), some difficulties still remain in monitoring brain tumor progression or treatment response in experimental and clinical approaches. For example, since the introduction of 2-deoxy-2-[ $\left[{ }^{18} \mathrm{~F}\right]$ fluoroD-glucose $\left({ }^{18} \mathrm{~F}\right.$-FDG)/PET, it has been widely used in clinical settings for evaluation of several malignancies, such as lung, colon, breast, head and neck and esophageal cancers (7). ${ }^{18} \mathrm{~F}-\mathrm{FDG}$ is metabolized as glucose but is stopped at the early step of metabolism, and can be used to detect the proliferation of tumor cells. Hence radiolabeled glucose analogs may serve as probes for molecular imaging of brain tumors. However, ${ }^{18} \mathrm{~F}-\mathrm{FDG}$ also accumulates in benign lesions, and false-positive results may occur during infection, inflammation and other therapy-related conditions (8). 3'-Deoxy-3'- $\left[{ }^{18} \mathrm{~F}\right]$ fluorothymidine $\left({ }^{18} \mathrm{~F}\right.$-FLT), a thymidine analog, often used in PET imaging for monitoring non-invasive cancer treatment, can be phosphorylated by thymidine kinase 1 (TK1) and trapped within tumor cells (9). It has been suggested that a multitracer approach using a combination of $\left[{ }^{18} \mathrm{~F}\right] \mathrm{FLT}$ and $\left[{ }^{18} \mathrm{~F}\right] \mathrm{FDG}$ may monitor early treatment response more 
effectively following cancer therapy (10). Other radiolabeled pyrimidine nucleoside derivatives, such as 2'-fluoro-2'-deoxy$\beta$-D-arabinofuranosyl-5-iodouracil (FIAU) labeled with radioiodine, 9-[4-[ $\left.{ }^{18} \mathrm{~F}\right]$ fluoro-3-(hydroxymethyl)butyl]guanine ([ $\left.\left.{ }^{18} \mathrm{~F}\right] \mathrm{FHBG}\right)$, and 9-[(1-[ $\left.{ }^{18} \mathrm{~F}\right]$ fluoro-3-hydroxy-2-propoxy) methyl]guanine $\left(\left[{ }^{18} \mathrm{~F}\right] \mathrm{FHPG}\right)$, have been demonstrated to accumulate selectively in HSV1-tk transfected cells, but significant non-specific phosphorylation is still noted $(11,12)$. On the other hand, 5-[ $\left.{ }^{76} \mathrm{Br}\right]$ bromo-2'-fluoro-2'-deoxyuridine $\left(\left[{ }^{76} \mathrm{Br}\right] \mathrm{BFU}\right)$ and 1-[2-deoxy-2- $\left[{ }^{18} \mathrm{~F}\right]$ fluoro- $\beta$-arabinofuranosyl5-bromouracil] ([$\left.\left.{ }^{18} \mathrm{~F}\right] \mathrm{FBAU}\right)$, both uracil analogs for DNA incorporation, have been developed for the imaging of tumor proliferation with superior specificity for the HSV1- $t k$ reporter system $(13,14)$. However, one disadvantage of $\left[{ }^{76} \mathrm{Br}\right]$ is that patients may be exposed to a higher radiation dose due to its long half-life and high energy (16.2 h, 3.6 MeV). The shorter half-life and optimal energy of $\left[{ }^{18} \mathrm{~F}\right](110 \mathrm{~min}, 0.64 \mathrm{MeV})$ makes $\left[{ }^{18} \mathrm{~F}\right]$-labeled pharmaceuticals more convenient for clinical PET imaging. It has been shown that $\left[{ }^{18} \mathrm{~F}\right]$-labeled proteins provide higher availability and better imaging quality (15). The advantages of $\left[{ }^{18} \mathrm{~F}\right]$-labeled FBAU and combined with PET imaging in cellular proliferation, neuroscience and oncology have been reported (16-18).

In our previous studies, 4-borono-2- $\left[{ }^{18} \mathrm{~F}\right]$-fluoro-L-phenylalanine-fructose ( $\left.\left[{ }^{18} \mathrm{~F}\right] \mathrm{FBPA}-\mathrm{Fr}\right)$ has been shown to have higher accumulation in tumors of F98 glioma-bearing rats, and can be used as a probe to mimic BPA-Fr in the treatment of brain tumors by boron neutron capture therapy (BNCT) (19). Furthermore, specific tumor accumulation with higher tumorto-normal brain ratio of $\left[{ }^{18} \mathrm{~F}\right] \mathrm{FBPA}-\mathrm{Fr}$ than that of $\left[{ }^{18} \mathrm{~F}\right] \mathrm{FDG}$ has also been demonstrated (20).

Establishment of a brain tumor-bearing animal model with a reporter gene-reporter probe (RGRP) system for multimodalities of molecular imaging may provide a useful platform for monitoring theranostics of tumor treatment (21-23), particularly for aggressive brain tumors such as GBM. The purpose of the present study was to investigate the potential application of $\left[{ }^{18} \mathrm{~F}\right] \mathrm{FBAU}$ as a PET reporter probe for monitoring tumor progression in an F98/tk-luc glioma-bearing rat model.

\section{Materials and methods}

Establishment of the pCl-tk-IRES-luc plasmid and the F98-tk-luc stable clone. The pC1-tk-IRES-luc plasmid was constructed according to our previous study (24). F98 rat glioma tumor cells were transfected with this plasmid using cationic polymer, jetPEI (Polyplus Transfection, Strasbourg, France). F98 cells were cultured in a 6 -well plate $\left(2 \times 10^{5}\right.$ cells/ well) after transfection. In a 1.5-ml Eppendorf vial, $3 \mu \mathrm{g}$ of DNA plasmid was mixed with $6 \mu \mathrm{l}$ jetPEI reagent in $100 \mu \mathrm{l}$ of $150 \mathrm{mM} \mathrm{NaCl}$ followed by gentle vortexing, and then briefly spun down. The mixtures were incubated for $30 \mathrm{~min}$ at room temperature and were added to the well. Transfection was performed at $37^{\circ} \mathrm{C}$ for $24 \mathrm{~h}$ in a humidified incubator with $5 \% \mathrm{CO}_{2}$. Stable clones were selected with $600 \mu \mathrm{g} / \mathrm{ml}$ of $\mathrm{G} 418$ (Calbiochem, Darmstadt, Germany).

Luciferase (luc) gene expression assayed with bioluminescent imaging (BLI). For the luc activity assay, the cell lysate was prepared by performing a freeze-thaw cycle in reporter lysis buffer (Promega, Madison, WI, USA). The bioluminescence was measured with a luminometer (Wallac 1420 Multilabel Counter; Life Sciences, Pittsburgh, PA, USA). The protein concentration of the cell lysate in each well was measured with a Bio-Rad protein assay kit (Bio-Rad Laboratories, Hercules, CA, USA) (24). F98 cells transfected with pC1-tk-IRES-luc vectors were cultured in a $6-\mathrm{cm}$ diameter dish for 14 days, and 30 colonies were selected. Each colony was cultured in a $3-\mathrm{cm}$ diameter dish for cell proliferation. Cells were treated with $200 \mu \mathrm{l}$ lysis buffer, and were frozen at $-80^{\circ} \mathrm{C}$ for $1 \mathrm{~h}$. After thawing and centrifugation (1,200 rpm, Kubota Laboratory Centrifuge, model 5100; Kubota Corp., Tokyo, Japan), the supernatant was transferred to a black 96 -well plate filled with D-luciferin. The bioluminescence was quantified as photons/ sec/mg. For BLI imaging in vivo, male Fischer 344 rats and NOD/SCID mice were anaesthetized with 1-3\% isofluorane, and then an i.p. injection with $300 \mu \mathrm{l}$ D-luciferin $(150 \mathrm{mg} / \mathrm{kg}$ in PBS) as the substrate. The IVIS 50 Imaging System (Xenogen, Alameda, CA, USA) was used for image acquisition. The time duration was $15 \mathrm{~min}$ for rats and $2 \mathrm{~min}$ for mice, followed by quantification with the Living Image software (Xenogen).

HSV1-tk gene expression assay. F98/tk-luc cells were cultured in 96-well plates $(3,000$ cells/200 $\mu \mathrm{l} /$ well $)$ for $24 \mathrm{~h}$, and then treated with 0-200 mM ganciclovir (GCV; F. Hoffmann-La Roche Ltd., Basel, Switzerland) for 120 h. 3-(4,5-Dimethylthiazol-2-yl)-2,5-diphenyltetrazolium bromide (MTT; Sigma-Aldrich, St. Louis, MO, USA) colorimetric assay was used to determine cell survival (24).

Radiosynthesis of $\left[{ }^{131} I\right] F I A U$ and $\left[{ }^{18} \mathrm{~F}\right] F B A U .\left[{ }^{131} \mathrm{I}\right] \mathrm{FIAU}$ was prepared according to our previous study (25). $\left[{ }^{18} \mathrm{~F}\right] \mathrm{FBAU}$ was synthesized according to a study reported by Alauddin et al (26). The purification of $\left[{ }^{18} \mathrm{~F}\right] \mathrm{FBAU}$ was followed according to our previous study (27).

$\left[{ }^{131}\right.$ I]FIAU cellular uptake assay. $\left[{ }^{131} \mathrm{I}\right] \mathrm{FIAU}\left(3.7 \times 10^{4} \mathrm{~Bq} / \mu \mathrm{l}\right)$ was added to each well $\left(1 \times 10^{6}\right.$ cells $/ 3 \mathrm{ml} /$ well in 6 -well culture plates), followed by incubation at $37^{\circ} \mathrm{C}$ for 15 and $30 \mathrm{~min}, 1,2$ and $4 \mathrm{~h}$, respectively. Triplicates were performed at each time point.

Cell culture, orthotropic F98/tk-luc glioma-bearing rat model and subcutaneous mouse tumor inoculation. F98 glioma cells (a kind gift from Dr Rolf F. Barth, The Ohio State University, USA) were transfected with the pC1-tk-IRES-luc plasmid (24), and renamed as F98/tk-luc glioma cells. These cell lines were cultured in Dulbecco's modified Eagle's medium (DMEM) (containing 10\% FBS, $100 \mathrm{U} / \mathrm{ml}$ penicillin and $100 \mu \mathrm{g} / \mathrm{ml}$ streptomycin) at $37^{\circ} \mathrm{C}$ in a humidified atmosphere of $5 \% \mathrm{CO}_{2}$. Male Fischer 344 rats (12-14 weeks of age, 250-280 g) were anesthetized intraperitoneally (i.p.) with a mixture of $110 \mathrm{mg} /$ $\mathrm{kg}$ ketamine and $9 \mathrm{mg} / \mathrm{kg}$ xylazine. F98/tk-luc glioma cells $\left(1 \times 10^{5}\right)$ in $10 \mu 1 \mathrm{Mg}^{2+} / \mathrm{Ca}^{2+}$-free Hanks' balanced salt solution (19) were then slowly $(15-20 \mathrm{sec})$ injected into the left brain region of the rats. The syringe was held still for 2 min after injection before withdrawing, and the injection hole was sealed with bone wax. Finally, the wound was flushed with iodinated alcohol and sutured with a sterilized steel clip. Male NOD/SCID mice were inoculated with $5 \times 10^{5}$ F98/tk-luc cells 


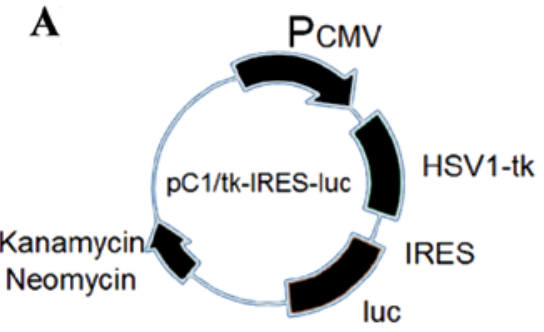

C

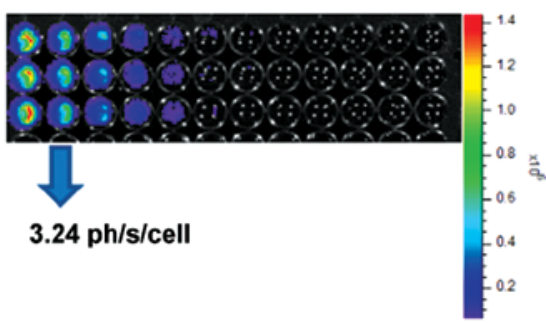

B

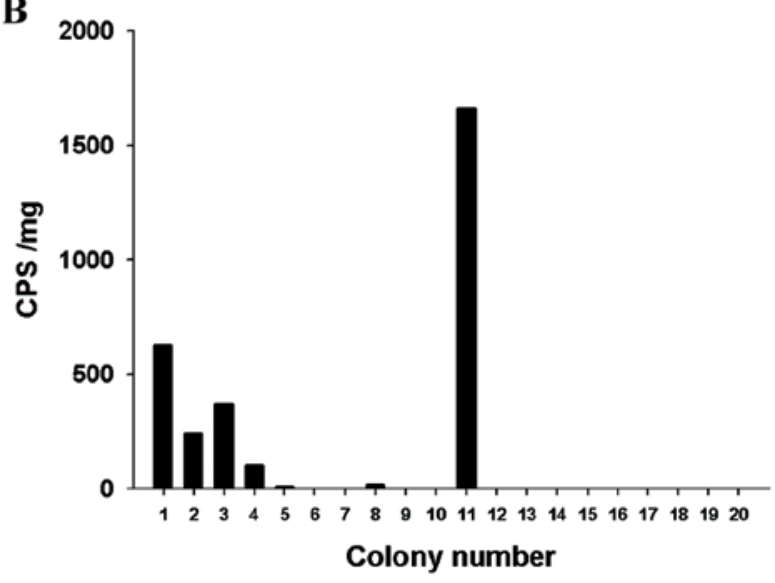

Figure 1. Bioluminescence assay of F98/tk-luc cells in vitro. (A) The genetic map of pCMV-tk-luc plasmid. (B) Luciferase expression assay of selected colonies was expressed in counts per second (CPS)/cell. The colony 11 with the highest value of light intensity was chosen for cell proliferation. (C) Cells $\left(1 \mathrm{x} 10^{5}\right)$ obtained from the expansion of colony 11 were serially diluted in a 96-well plate for assay of the luciferase activity. D-luciferin was added to each well and the plate was imaged for $1 \mathrm{~min}$. The expression of luciferase was 3.24 photons/sec/cell.

in $200 \mu 1$ serum-free medium subcutaneously in the right flank. The tumor volume was measured with a digital caliper.

Tumor volume assayed by MR imaging. 3T MR scanning was performed within $24 \mathrm{~h}$ post BLI imaging. A $40-\mathrm{mm}$ diameter field-of-view (FOV) orbital surface coil was used as the receiver (28). Each rat was anesthetized with 3-4 ml 10\% chloride hydrate. To obtain accurate position of the brain slice, the coronal and transverse plane scout images were scanned first. 2-4 coronal T1-weighted slices with a 1.5-mm thickness would cover the tumor. The T1-weighted images were acquired with a 256x 256 matrix, FOV=89 $\mathrm{mm}$, repetition time $=435 \mathrm{msec}$ and echo time $=12 \mathrm{msec}$. For contrast-enhanced images, each animal received $0.2 \mathrm{mmol} / \mathrm{kg}$ contrast medium i.v. $5 \mathrm{~min}$ prior to T1-weighted sequence imaging. T2-weighted images were acquired with a 256x256 matrix, FOV=89 mm, repetition time $=5,000 \mathrm{msec}$ and echo time $=104 \mathrm{msec}$. The DICOM images were collected, and the tumor boundary visualized in each slice was defined as the region-of-interest (ROI) using ImageJ (National Institutes of Health, Bethesda, MD, USA) for calculation. The sum of the ROI areas was processed by slice thickness to obtain the tumor volume.

Pathology examination. After examination, the rats were sacrificed with $\mathrm{CO}_{2}$. The brains were removed and fixed with $4 \%$ paraformaldehyde. The sections $(5-\mu \mathrm{m})$ obtained from the paraffin-embedded samples were stained with hematoxylin and eosin (H\&E).

Biodistribution of $\left[{ }^{18} \mathrm{~F}\right] \mathrm{FBAU}$ in the F98/tk-luc glioma-bearing rats. Biodistribution of $\left[{ }^{18} \mathrm{~F}\right] \mathrm{FBAU}$ was determined on the 13 th day post tumor implantation as previously described (19). Each rat was anesthetized with $3-4 \mathrm{ml}$ of $10 \%$ chloride hydrate. The F98/tk-luc glioma-bearing rats were injected with
$15 \mathrm{MBq} / 0.2 \mathrm{ml}\left[{ }^{18} \mathrm{~F}\right] \mathrm{FBAU}$ via lateral tail veins. At $0.5,1,1.5$ and $2 \mathrm{~h}$ post injection, the rats ( $\mathrm{n}=5$ per group) were sacrificed with $\mathrm{CO}_{2}$. Left and right brains, tumors, intestine, spleen, heart, blood, lung, liver, stomach, pancreas, kidneys, bone and muscles were removed, and the radioactivity was measured in a well-type multichannel analyzer (Raytest, Straubenhardt, Germany) (27). The uptake of $\left[{ }^{18} \mathrm{~F}\right] \mathrm{FBAU}$ in tissues or organs was expressed in counts per second (cps) corrected with the decay, and was normalized as the percentage injected dose per gram of tissue $(\% \mathrm{ID} / \mathrm{g})$.

PET scanning. The PET images of F98/tk-luc glioma-bearing rats i.v. injected with $50 \mathrm{MBq} / 0.1 \mathrm{ml}\left[{ }^{18} \mathrm{~F}\right] \mathrm{FBAU}$ were acquired up to $160 \mathrm{~min}$ using the PET/CT system (GE Discovery LS PET-CT; GE Healthcare, Cleveland, OH, USA) on day 13 post tumor implantation. The imaging parameters were set as follows: $2-\mathrm{D}$ brain model, $\mathrm{FOV}=15.52 \mathrm{~cm}$, spatial resolution $=5.46 \mathrm{~mm}, 128 \times 128$ matrix size. The reconstruction algorithm using ordered subset expectation maximization (OSEM) was similar to that of a previous study (19). The images were acquired with axial, coronal and sagittal planes, respectively. Each rat was anesthetized with 3-4 ml 10\% chloride hydrate and i.v. injected with $0.1 \mathrm{ml}$ of $50 \mathrm{MBq}\left[{ }^{18} \mathrm{~F}\right]$ FBAU. Data acquisition by PET scanning was initiated at the first minute after drug injection. Dynamic coronal and sagittal images were acquired using 1060 -sec frames and 10 2-min frames, followed by 10 -min frames up to $2 \mathrm{~h}$. Standardized uptake values (SUVs) were calculated and normalized to body weight (BW) (29). \%SUV was calculated using the following formula: \%SUV = [(left brain SUV - right brain SUV $) /$ right brain SUV] x $100 \%$.

Ex vivo autoradiography. The ex vivo autoradiography was performed according to a previous report (20). Fischer 344 

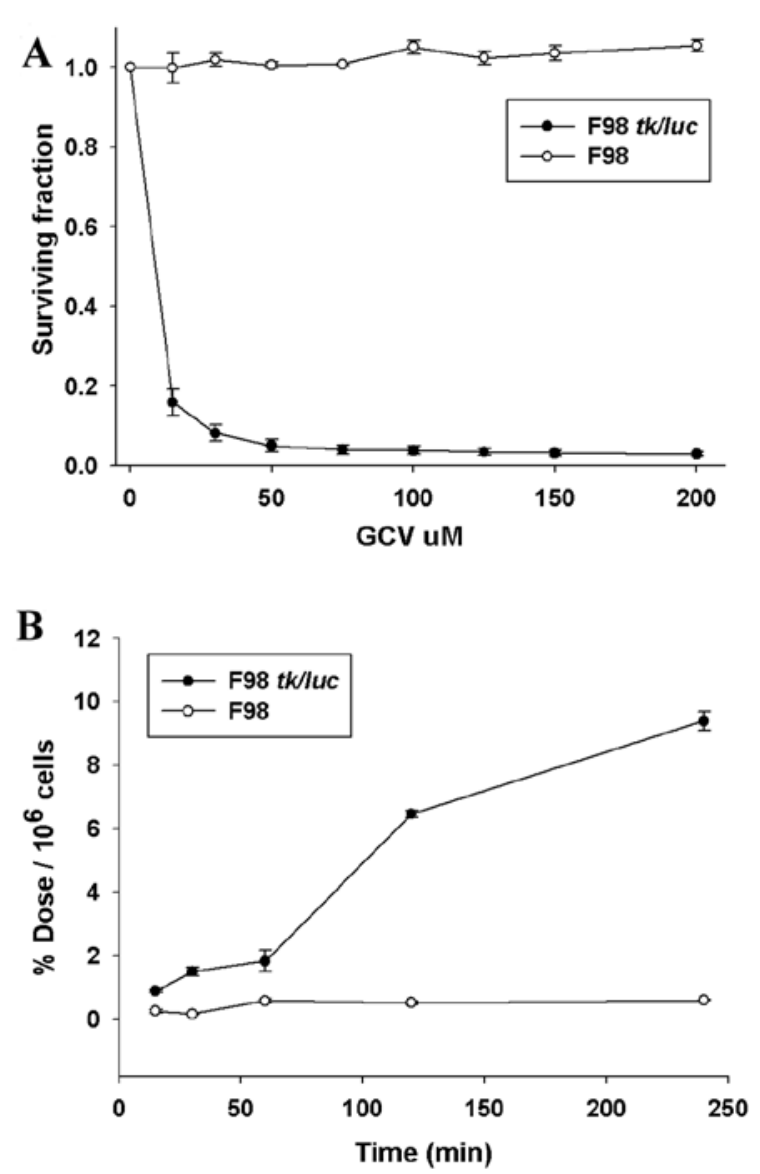

Figure 2. HSV1-tk reporter gene assay of F98/tk-luc cells in vitro. (A) The surviving fractions of F98/tk-luc cells that were decreased to 0.2 or less after treatment with various concentrations of $\mathrm{GCV}(\mathrm{n}=6)$. (B) Cellular uptake was determined by $\gamma$-counter after $\left.{ }^{131} \mathrm{I}\right] \mathrm{FIAU}$ addition. The uptake of F98/tk-luc cells increased to $\sim 10 \%$ dose $/ 10^{6}$ cells after $240 \min (n=5)$.

rats were anesthetized with 1-3\% isofluorane. At 30,60,90 and 120 min post i.v. injection of $\left[{ }^{18} \mathrm{~F}\right] \mathrm{FBAU}(15-20 \mathrm{MBq} / 0.7 \mathrm{ml})$, the rats were sacrificed with $\mathrm{CO}_{2}$. The brains were removed and embedded with OCT on a cryostat holder (diameter $2.7 \mathrm{~cm}$ ), and were frozen immediately at $-80^{\circ} \mathrm{C}$. Coronal sections (20- $\mu \mathrm{m}$ thick) were performed using a cryomicrotome (Leica CM3050; Leica Microsystems, Bensheim, Germany). The imaging plates were assayed with an FLA5000 reader (Fuji Photo Film Co., Ltd., Tokyo, Japan) with the following settings: $\mathrm{PMT}=800 \mathrm{~V}$, resolution $=50 \mu \mathrm{m}$, gradation $=16$ bits, dynamic range $=\mathrm{L} 5$ and sensitivity $=5,000$ to acquire the phosphor images. ROIs were circled along the tumor contour of the F98/tk-luc glioma, and ROIs of equal size in the normal brain region at corresponding brain position were used as the reference. The intensity of photo-stimulated luminescence (PSL) of the tumor and normal brain tissue was measured with Image Gauge (version 4.0, Science Lab 2001; Fuji Photo Film).

Statistical analysis. The Studen't t-test was used to analyze the significant difference between the control and drug-treated rats. All data are shown as the means \pm standard error (SE). Differences between the means were considered significant at $\mathrm{P} \leq 0.05$. The correlation between tumor volume and BLI photon flux was performed by Pearson correlation.
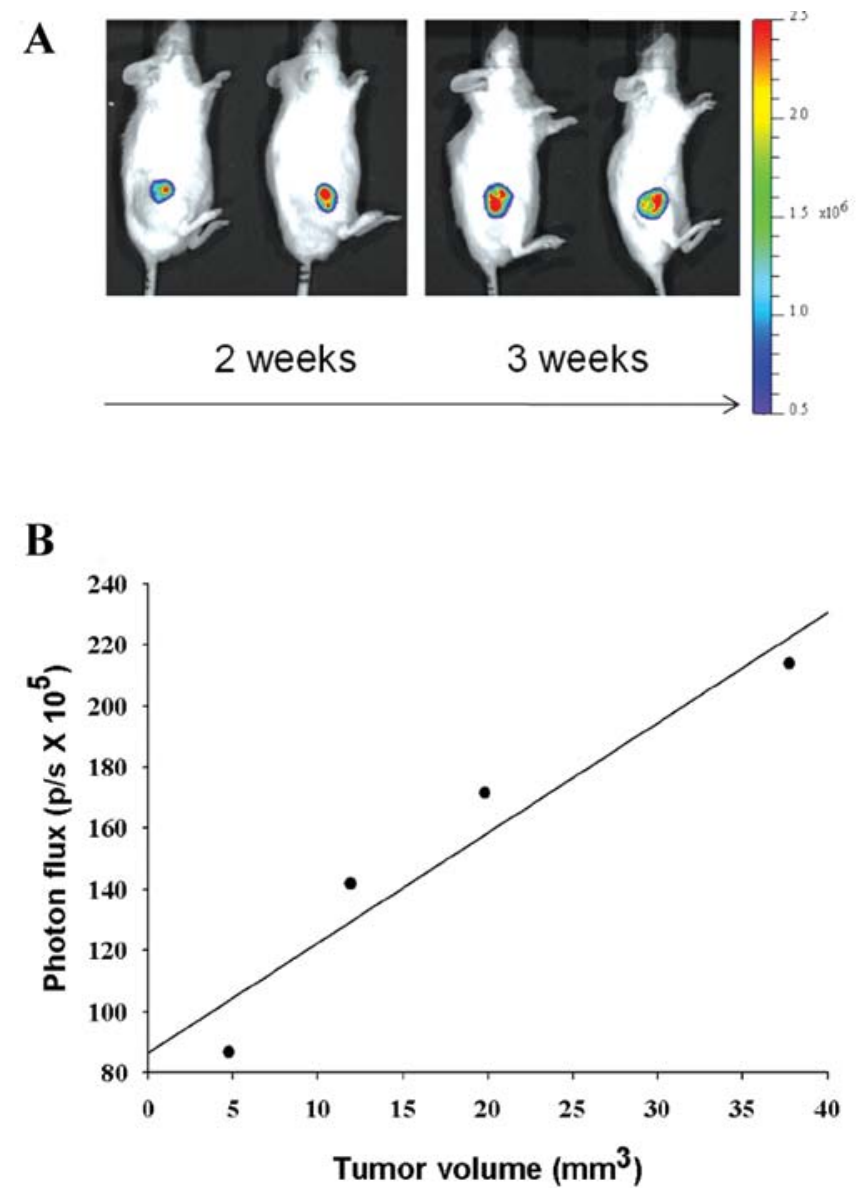

Figure 3. Luc expression of F98/tk-luc glioma in NOD/SCID mice. (A) The increasing luminescence of $\mathrm{F} 98 / \mathrm{tk}$-luc tumors in mice was consistent with the increasing size of the tumors measured with a caliper over 2-3 weeks post inoculation. (B) Linear plot indicating the correlation of luminescence with increasing tumor volume $\left(\mathrm{r}^{2}=0.908, \mathrm{P}<0.05, \mathrm{n}=4\right)$.

Table I. The tumor volumes assayed by 3T MR and photon flux rates of regions-of-interest (ROI) obtained from BLI of the rat brains were compared.

\begin{tabular}{lcc}
\hline Rat ID & $\begin{array}{c}\text { Tumor volume } \\
\left(\mathrm{mm}^{3}\right) \text { assayed } \\
\text { by MR }\end{array}$ & $\begin{array}{c}\text { Total photon flux rate } \\
\left(\text { photons/sec, } x ~ 10^{5}\right) \\
\text { obtained from BLI }\end{array}$ \\
\hline 1 & 1.93 & 4.38 \\
2 & 5.07 & 4.56 \\
3 & 8.62 & 9.78 \\
4 & 8.94 & 5.51 \\
5 & 52.77 & 41.40 \\
6 & 126.90 & 41.00 \\
7 & 213.0 & 58.90 \\
\hline
\end{tabular}

\section{Results}

Selection of stable clones and luc gene expression assay. The pC1-tk-IRES-luc vectors as shown in Fig. 1A were transfected into the F98 cell line, which was renamed F98/tk-luc. The colony exhibiting $l u c$ expression of $>1,500$ counts per sec 
A Total flux rate (photons/sec)
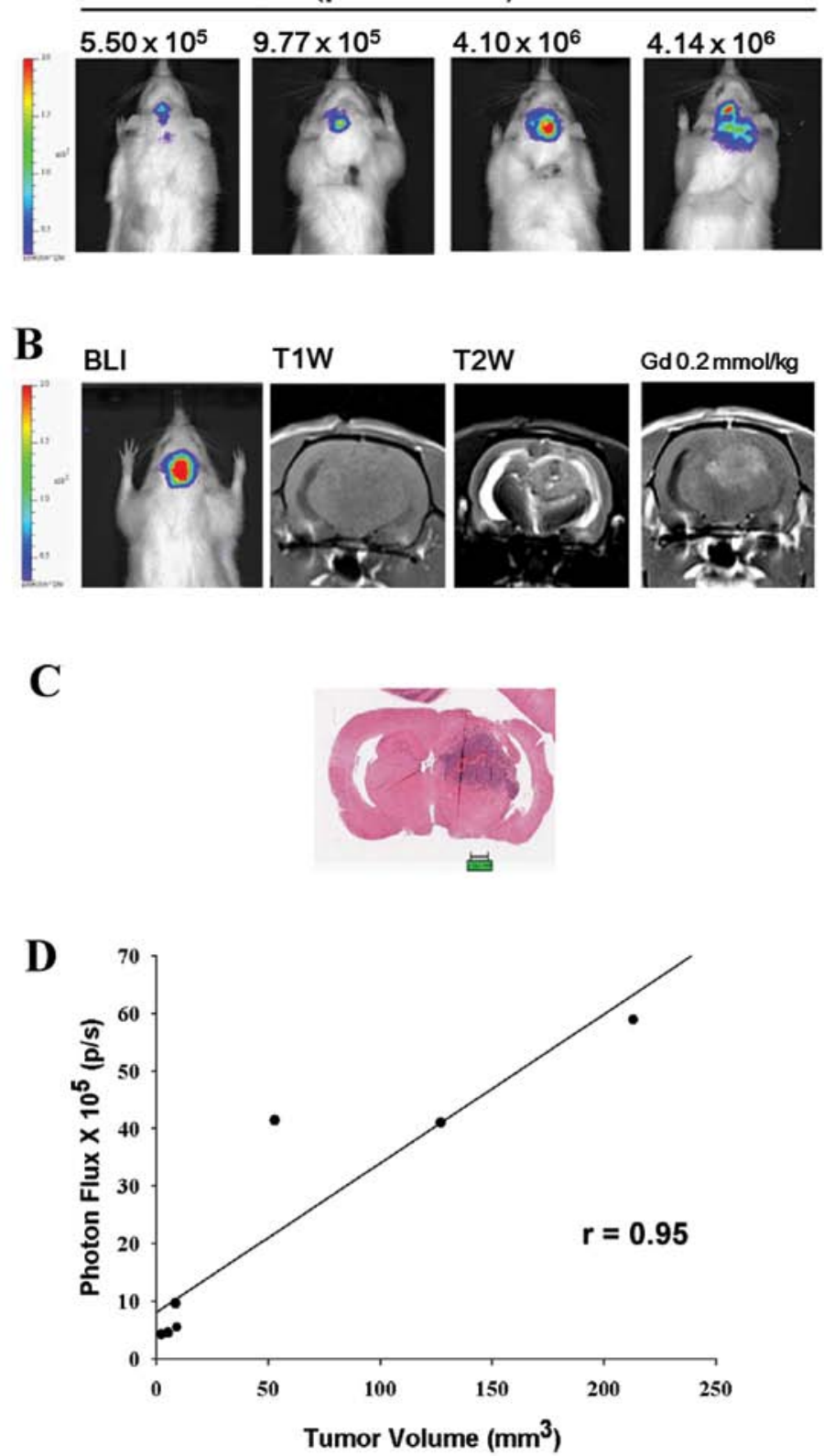

Figure 4. Brain images of Fischer 344 rats obtained by bioluminescent imaging (BLI), MR and histology. (A) BLI. (B) MR images by T1- and T2-weighed and post gadolinium $(0.2 \mathrm{mmol} / \mathrm{kg}$ ) injection compared to BLI (left panel) in a representative mouse. (C) Histopathology of a brain section stained with H\&E (scale bar, $1.01 \mathrm{~mm}$ ). (D) Tumor volume estimated from photon flux rates of ROIs by BLI are correlated with those calculated from MR images ( $\mathrm{r}=0.95$ ).

per $\mathrm{mg}(\mathrm{cps} / \mathrm{mg})$ was selected for cell expansion as shown in Fig. 1B (colony no. 11). The bioluminescence of luc gene expression in the F98/tk-luc cells was 3.24 photons/sec/cell (Fig. 1C).

Expression of thymidine kinase (tk). F98/tk-luc cells were treated with various concentrations of ganciclovir (GCV). The surviving fraction (SF) of F98/tk-luc cells was $18 \%$ at $15 \mu \mathrm{M}$ and remained $\sim 100 \%$ up to $200 \mu \mathrm{M}$ for parental F98 cells after GCV treatment (Fig. 2A). In addition, the uptake of $\left[{ }^{131} \mathrm{I}\right]$ FIAU in the F98/tk-luc cells was increased with time, but was not found in the parental F98 cells (Fig. 2B).

Tumor growth monitored using bioluminescent imaging (BLI) in F98/tk-luc tumor-bearing mice. F98/tk-luc cells were inoculated subcutaneously into the right flank of NOD/SCID mice. Two weeks later, tumor growth was monitored with BLI as shown in Fig. 3A. The quantification of bioluminescence (photons/sec x 10 $0^{5}$ ) vs. tumor volume $\left(\mathrm{mm}^{3} \times 10\right)$ from representative mice is plotted in Fig. 3B with linear regression $\left(r^{2}=0.90\right)$.

Bioluminescent imaging (BLI), MR imaging and histopathology. The BLI of F98/tk-luc glioma-bearing rats was obtained using the IVIS50 optical imaging system (Xenogen Corp.) on day 13 post inoculation of F98-tk-luc glioma cells into the left brain. The total photon flux rates of the tumors were $5.50 \times 10^{5}-4.14 \times 10^{6}$ as shown in Fig. 4 A (images were from representative rats). MR images of the representative rat brains were obtained from a 3T MR scanner including T1-, 

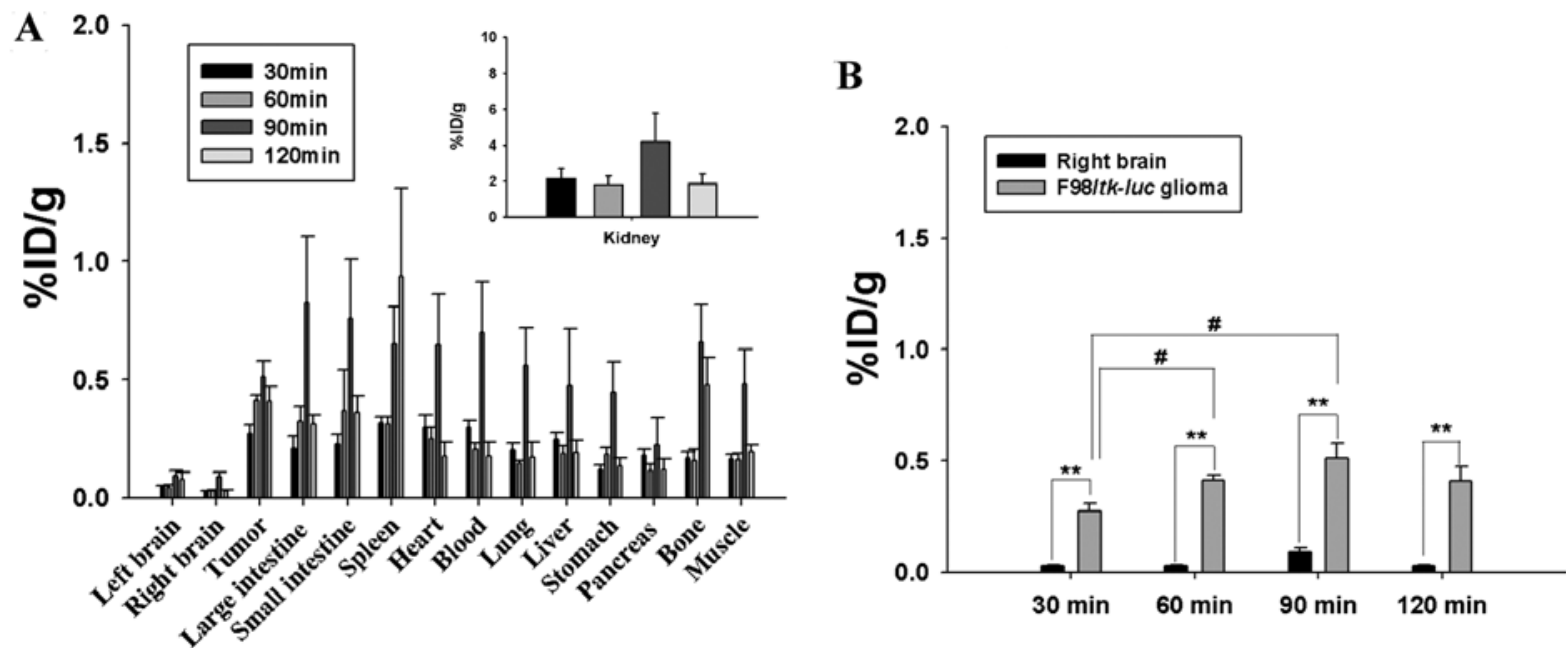

Figure 5. Biodistribution of $\left.{ }^{18} \mathrm{~F}\right] \mathrm{FBAU}$ in F98/tk-luc tumor-bearing rats $(\mathrm{n}=5)$. (A) Specific radioactivities (\%ID/g) in the left and right brain, intestine, spleen, heart, kidney, lung, liver, stomach, pancreas, bone, muscle, blood and tumor at various time points are shown. (B) The ratios calculated from \%ID/g of $\left[{ }^{18} \mathrm{~F}\right] \mathrm{FBAU}$ in F98/tk-luc glioma-to-normal right brain at 30,60, 90 and $120 \mathrm{~min}$ post injection of $\left[{ }^{18} \mathrm{~F}\right] \mathrm{FBAU}$ were $9.16,14.24,5.70$ and 13.70 , respectively. The $\% \mathrm{ID} / \mathrm{g}$ of $\left[{ }^{18} \mathrm{~F}\right] \mathrm{FBAU}$ in the F98/tk-luc glioma was significantly higher than that of the right brain $\left({ }^{* *} \mathrm{P}<0.005\right)$. In addition, \%ID/g values for the 60 and $90 \mathrm{~min}$ groups were also significantly higher than that of the 30 min post injection group $\left({ }^{\#} \mathrm{P}<0.05\right)$.

Table II. Biodistribution of $\left[{ }^{18} \mathrm{~F}\right] \mathrm{FBAU}$ in various tissues/organs of the F98/tk-luc glioma-bearing Fischer 344 rats after intravenous injection $(\mathrm{n}=5$; mean $\pm \mathrm{SE})$.

Uptake (\%ID/g)

\begin{tabular}{lcccc}
\cline { 2 - 4 } Tissues/organs & $30 \mathrm{~min}$ & $60 \mathrm{~min}$ & $90 \mathrm{~min}$ & $120 \mathrm{~min}$ \\
\hline Left brain & $0.048 \pm 0.004$ & $0.047 \pm 0.008$ & $0.094 \pm 0.023$ & $0.081 \pm 0.028$ \\
Right brain & $0.030 \pm 0.001$ & $0.029 \pm 0.004$ & $0.090 \pm 0.019$ & $0.030 \pm 0.005$ \\
Tumor & $0.275 \pm 0.034$ & $0.413 \pm 0.023$ & $0.513 \pm 0.066$ & $0.411 \pm 0.061$ \\
Large intestine & $0.212 \pm 0.051$ & $0.325 \pm 0.060$ & $0.826 \pm 0.282$ & $0.311 \pm 0.040$ \\
Small intestine & $0.231 \pm 0.039$ & $0.368 \pm 0.175$ & $0.759 \pm 0.248$ & $0.362 \pm 0.071$ \\
Spleen & $0.315 \pm 0.025$ & $0.311 \pm 0.030$ & $0.650 \pm 0.158$ & $0.936 \pm 0.375$ \\
Heart & $0.296 \pm 0.054$ & $0.252 \pm 0.044$ & $0.648 \pm 0.212$ & $0.176 \pm 0.059$ \\
Blood & $0.296 \pm 0.032$ & $0.207 \pm 0.027$ & $0.699 \pm 0.217$ & $0.178 \pm 0.058$ \\
Lung & $0.203 \pm 0.030$ & $0.146 \pm 0.012$ & $0.562 \pm 0.158$ & $0.172 \pm 0.066$ \\
Liver & $0.248 \pm 0.031$ & $0.189 \pm 0.033$ & $0.476 \pm 0.240$ & $0.191 \pm 0.053$ \\
Stomach & $0.120 \pm 0.017$ & $0.186 \pm 0.028$ & $0.448 \pm 0.127$ & $0.136 \pm 0.031$ \\
Pancreas & $0.179 \pm 0.027$ & $0.117 \pm 0.026$ & $0.227 \pm 0.112$ & $0.120 \pm 0.046$ \\
Kidney & $2.151 \pm 0.544$ & $1.822 \pm 0.480$ & $4.183 \pm 1.604$ & $1.868 \pm 0.550$ \\
Bone & $0.170 \pm 0.026$ & $0.159 \pm 0.046$ & $0.657 \pm 0.160$ & $0.479 \pm 0.116$ \\
Muscle & $0.165 \pm 0.019$ & $0.161 \pm 0.026$ & $0.484 \pm 0.143$ & $0.195 \pm 0.031$ \\
Tumor/right brain & 9.16 & 14.24 & 5.70 & 13.70 \\
\hline
\end{tabular}

T2-weighted and contrast-enhanced images ( $\mathrm{Gd} 0.2 \mathrm{mmol} / \mathrm{kg}$ ) on day 14 (Fig. 4B).

In the present study, the tumor volume and its extent were not able to be demonstrated well by T1-weighted images but were greatly enhanced post contrast injection, which showed a clear boundary between the tumor and normal brain tissue. On the other hand, the tumor location could be shown by T2-weighted images whereas the boundary between the tumor and normal tissue was still indistinguishable. Fischer 344 rats after MR scanning were sacrificed, and the brain tissues were removed and preserved in $4 \%$ paraformaldehyde. H\&E staining was subsequently performed. The results are shown in Fig. $4 \mathrm{C}$, in which the tumor location and tumor volume were the same as those of the MR images shown in Fig. 4B.

The total photon fluxes of F98-tk-luc tumors obtained from BLI vs. tumor volumes calculated by 3T MR contrast- 
A

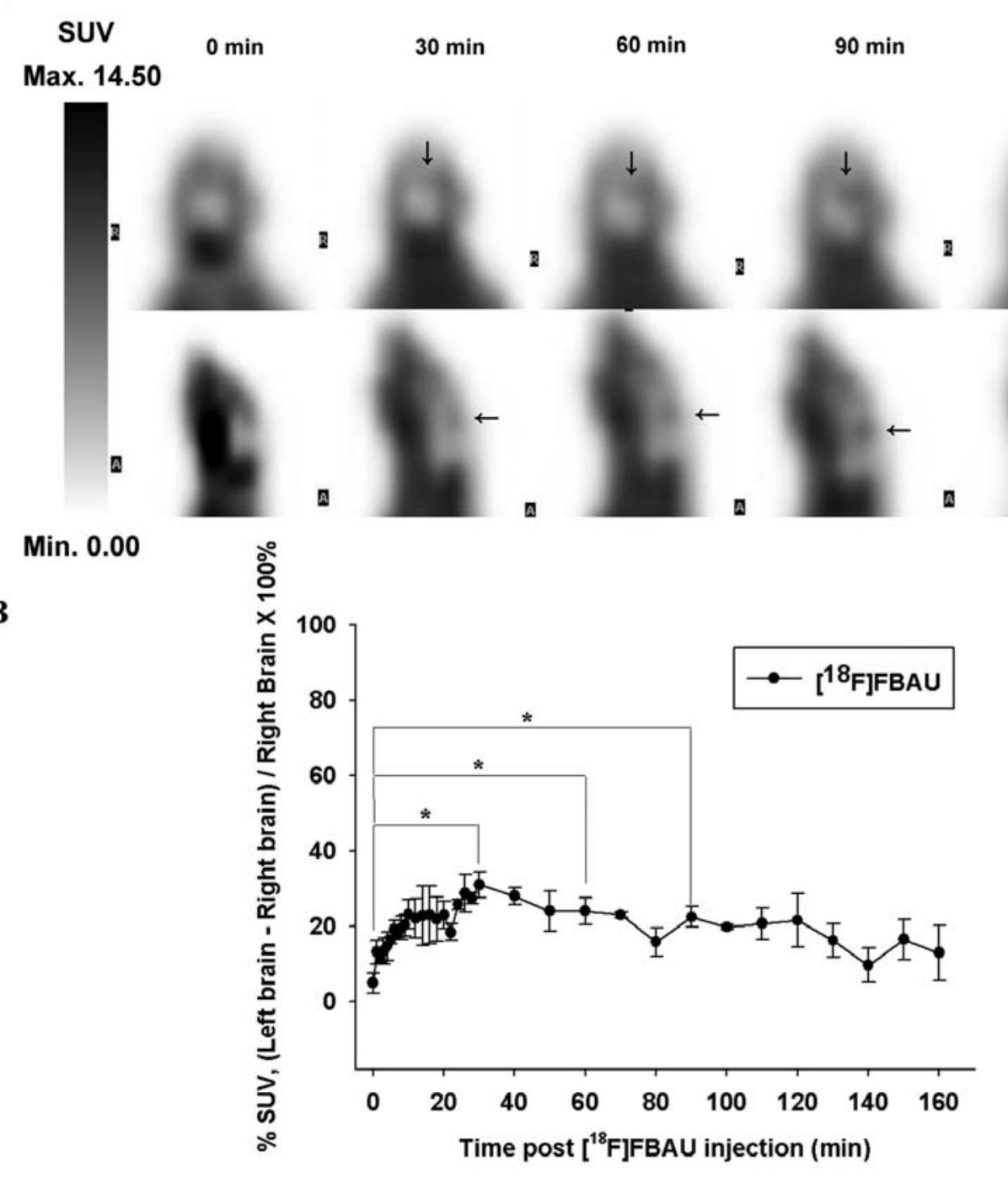

Figure 6. The PET images and \%SUV of F98/tk-luc glioma-bearing Fischer 344 male rats at various time points post intravenous injection of $\left[{ }^{18} \mathrm{~F}\right] \mathrm{FBAU}$ $(50 \mathrm{MBq} / 0.2 \mathrm{ml})$. (A) PET image of F98/tk-luc glioma is indicated by an arrow in the image of the left brain. (B) The \%SUVs of [ $\left.{ }^{18} \mathrm{~F}\right] \mathrm{FBAU}$ at 30,60 and $90 \mathrm{~min}$ post injection of $\left[{ }^{18} \mathrm{~F}\right] \mathrm{FBAU}$ were significantly higher than that of the on-set time point post injection by $9-20 \%\left({ }^{*} \mathrm{P}<0.05\right.$; data are expressed as mean $\left.\pm \mathrm{SE}, \mathrm{n}=5\right)$.

enhanced images are tabulated in Table I and plotted in Fig. 4D. The Pearson correlation coefficient (r) is 0.95 .

Biodistribution of ${ }^{18} \mathrm{~F}-\mathrm{FBAU}$ in the F98/tk-luc tumorbearing rats. The accumulation of $\left[{ }^{18} \mathrm{~F}\right] \mathrm{FBAU}$ in tissues of the F98/tk-luc tumor-bearing Fischer 344 rats $(\mathrm{n}=5)$ was measured at different time points after administration of $15 \mathrm{MBq} / 0.2 \mathrm{ml}$. The highest uptake of $\left[{ }^{18} \mathrm{~F}\right] \mathrm{FBAU}$ was found in kidneys, suggesting that the elimination route of $\left[{ }^{18} \mathrm{~F}\right] \mathrm{FBAU}$ is via renal clearance. The uptake of $\left[{ }^{18} \mathrm{~F}\right] \mathrm{FBAU}$ in the left and right normal brain tissues was very low in the range of 0.03-0.09\%ID/g (Fig. 5A and Table II). Notably, the uptake of $\left[{ }^{18} \mathrm{~F}\right] \mathrm{FBAU}$ in the F98/tk-luc glioma tumors reached up to $0.51 \pm 0.06$ at $90 \mathrm{~min}$ post injection (Fig. 5B). The uptake of $\left[{ }^{18} \mathrm{~F}\right] \mathrm{FBAU}$ in the tumors was significantly higher than those of normal brain tissues $(\mathrm{P}<0.01)$. In addition, the uptake of $\left[{ }^{18} \mathrm{~F}\right] \mathrm{FBAU}$ in tumors in the 60 and $90 \mathrm{~min}$ post-injection groups was significantly higher than that of the 30 min postinjection group $(\mathrm{P}<0.05)$.
PET images of ${ }^{18} \mathrm{~F}$-FBAU in the F98/tk-luc tumor-bearing rat model. The coronal and sagittal PET images of the F98/tk-luc glioma-bearing rats were imaged with a clinical PET. The result showed that accumulation of $\left[{ }^{18} \mathrm{~F}\right] \mathrm{FBAU}$ was noted in F98/tk-luc glioma in the brain (arrows in Fig. 6A). The $\left[{ }^{18} \mathrm{~F}\right]$ FBAU/PET images at 30-90 min post injection showed significantly higher \%SUV values than that acquired at the initial time point $(\mathrm{P}<0.005$; Fig. 6B).

Ex vivo autoradiography. Biodistribution of $\left[{ }^{18} \mathrm{~F}\right] \mathrm{FBAU}$ in the rat brain was also examined by ex vivo autoradiography. The result showed that the uptake of $\left[{ }^{18} \mathrm{~F}\right] \mathrm{FBAU}$ in the F98/tk-luc gliomas reached the maximum value at 90 min post injection (Fig. 7A). Autoradiogram of $\left[{ }^{18} \mathrm{~F}\right] \mathrm{FBAU}$ in the F98/tk-luc gliomas showed a similar result as that of the biodistribution study with the highest uptake at $90 \mathrm{~min}$ post injection. The quantification of the autoradiogram is depicted in Fig. 7B. The uptake of $\left[{ }^{18} \mathrm{~F}\right] \mathrm{FBAU}$ assayed by BLI in units of photostimulated luminescence (PSL) $/ \mathrm{mm}^{2}$ in the F98/tk-luc gliomas 
A

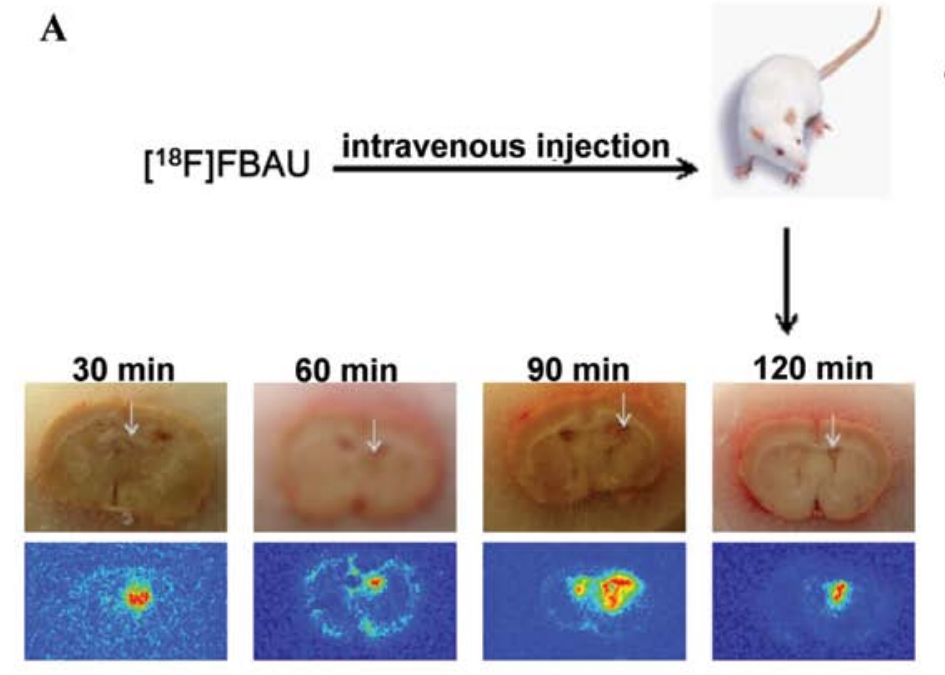

B

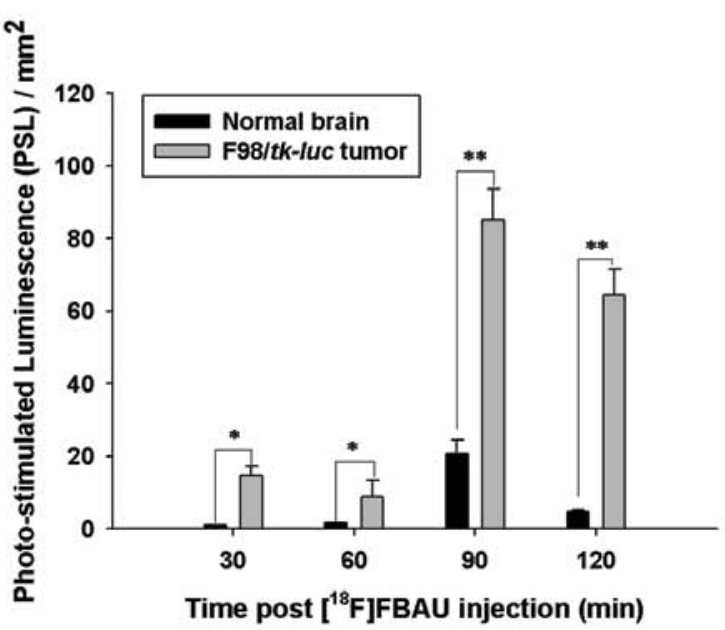

Figure 7. Ex vivo autoradiography. (A) Brain autoradiograms of the F98/tk-luc glioma-bearing Fischer 344 male rats after intravenous injection of $20 \mathrm{MBq} / 0.2 \mathrm{ml}$ $\left[{ }^{18} \mathrm{~F}\right] \mathrm{FBAU}$. (B) Relative optical intensity $\left(\mathrm{PSL} / \mathrm{mm}^{2}\right)$ of tumors and normal brain derived from autoradiograms $\left({ }^{*} \mathrm{P}<0.05\right.$, ${ }^{* *} \mathrm{P}<0.005$; data were expressed as mean $\pm \mathrm{SE}, \mathrm{n}=5$ ).

was significantly higher at each time point from 30 to $120 \mathrm{~min}$ compared to that of the contralateral normal brain tissue (Fig. 7B).

\section{Discussion}

[24I]FIAU has been reported to be the first PET probe used in the HSV1-tk reporter gene expression system $(11,30)$. Although FIAU is preferentially phosphorylated by HSV1-thymidine kinase (TK) compared to mammalian TK, a certain amount of FIAU is accumulated in cells which do not express HSV1-TK resulting in non-specific signals (31). It has been reported that no difference in biodistribution exists between $\left[{ }^{14} \mathrm{C}\right] \mathrm{FIAU}$ and $\left[{ }^{76} \mathrm{Br}\right] \mathrm{FBAU}$; the uptake of the latter, however, is constantly higher than that of $\left[{ }^{14} \mathrm{C}\right] \mathrm{FIAU}$ in RG2-TK rat glioma cells. Furthermore, the uptake of $\left[{ }^{76} \mathrm{Br}\right] \mathrm{FBAU}$ is not affected significantly by endogenous mammalian thymidine kinase (14). 2'-Deoxy-2'-[18F]fluoro-5-ethyl-1- $\beta$-D-arabinofuranosyluracil $\left(\left[{ }^{18} \mathrm{~F}\right] \mathrm{FEAU}\right)$, another pyrimidine nucleoside analog, has been shown to have higher selectivity and affinity for HSV- $t k$ in subcutaneous RG2-TK ${ }^{+}$tumors and C6-tk cells compared to other radiotracers $(32,33)$, but thorough evaluation by other modalities is lacking. In the present study, we demonstrated the tumor-specific radiotracing activity of $\left[{ }^{18} \mathrm{~F}\right] \mathrm{FBAU}$ in an F98/tk-luc glioma-bearing animal model using HSV1-tk reporter gene $/\left[{ }^{18} \mathrm{~F}\right] \mathrm{FBAU}$ reporter probe system combined with multimodalities of molecular imaging with PET, BLI, MRI and $e x$ vivo autoradiography. $\left[{ }^{18} \mathrm{~F}\right] \mathrm{FBAU}$ showed better differentiation with higher uptake in the tumors compared to the contralateral normal brain in the F98/tk-luc gliomabearing animal model between 30 to 120 min post injection (Figs. 5B and 6B). Both PET imaging and ex vivo autoradiography also showed the persistent accumulation of $\left[{ }^{18} \mathrm{~F}\right] \mathrm{FBAU}$ in the F98/tk-luc glioma-bearing animal model from 90 to 120 min post injection (Figs. 6A and 7B), which was $\sim 5-14$ times higher than the other radiotracers, such as $\left[{ }^{76} \mathrm{Br}\right] \mathrm{FBAU}$, at $120 \mathrm{~min}$ post injection as reported in other studies (14). The results suggest that $\left[{ }^{18} \mathrm{~F}\right] \mathrm{FBAU}$ is a suitable reporter probe for the imaging of HSV1-tk expression.

For the purpose of tracing tumor growth progression, molecular imaging techniques other than traditional caliper measurement have been developed. A statistical correlation was developed to correlate the relationship between anatomical size and bioluminescent intensity collected from ROI of the tumors in a brain-tumor bearing animal model (23). Notably, the brain tumor volume assayed by bioluminescent imaging (BLI) has been reported to poorly correlate with images obtained from MRI $(34,35)$. Nevertheless, metastatic breast cancer in a rat model monitored using MRI at 3 tesla was found to correlate well with that by BLI (36). Although $\left[{ }^{18} \mathrm{~F}\right] \mathrm{FDG} / \mathrm{PET}$ imaging has been used for diagnosis of tumors as well as the malfunction of the brain, the uptake of $\left[{ }^{18} \mathrm{~F}\right] \mathrm{FDG}$ remains high in normal brain tissues, causing difficulties in differentiating normal brain from malignancy $(20,37)$. In order to monitor the progression of brain tumor growth in the glioma-bearing animal model, we found a good correlation for the tumor volume acquired from MRI vs. that obtained from BLI in the F98/tk-luc glioma-bearing rat model (Fig. 4). The SUV of $\left[{ }^{18} \mathrm{~F}\right] \mathrm{FBAU}$ in F98/tk-luc glioma was $20 \%$ higher than that of contralateral normal brain tissue (Fig. 6). Hence $\left[{ }^{18} \mathrm{~F}\right] \mathrm{FBAU}$ may provide another advantage in brain tumor diagnosis and disease monitoring. As a result, this approach may have potential for using $\left[{ }^{18} \mathrm{~F}\right] \mathrm{FBAU}$ as a molecular probe combined with a reporter gene system, such as HSV1-tk, to monitor tumor growth progression in preclinical or clinical applications.

In conclusion, the uptake of $\left[{ }^{18} \mathrm{~F}\right] \mathrm{FBAU}$ and SUV of $\left[{ }^{18} \mathrm{~F}\right] \mathrm{FBAU}$ in brain tumors of an F98/tk-luc glioma-bearing rat model was significantly higher than that of normal brain tissues determined by biodistribution and PET, respectively. Both the volume and progression of tumors determined with MRI and BLI were well correlated in the F98/tk-luc glioma-bearing rat model using $\left[{ }^{18} \mathrm{~F}\right] \mathrm{FBAU}$ as a reporter probe. Radiolabeled FBAU combined with multimodalities of molecular imaging 
may also provide potential benefits for the evaluation of therapeutic efficacy of newly developed drugs against other brain diseases.

\section{Acknowledgements}

Thepresentstudy wassupportedby grantNSC92-2745-P-010-002 from the National Science Council, Taipei, Taiwan. We thank the staff of the Department of Radiopharmaceutical Production, Buddhist Tzu Chi General Hospital, Hualien, Taiwan for providing PET/CT imaging and advice for the synthesis of $\left[{ }^{18} \mathrm{~F}\right]$ FBAU. We also thank the Taiwan Mouse Clinic which is funded by the National Research Program for Biopharmaceuticals (NRPB) at the National Science Council (NSC) of Taiwan for technical support with the bioluminescent imaging experiment.

\section{References}

1. Stupp R, Mason WP, van den Bent MJ, et al: Radiotherapy plus concomitant and adjuvant temozolomide for glioblastoma. N Engl J Med 352: 987-996, 2005.

2. Sathornsumetee S and Rich JN: Designer therapies for glioblastoma multiforme. Ann NY Acad Sci 1142: 108-132, 2008.

3. Clarke J, Butowski N and Chang S: Recent advances in therapy for glioblastoma. Arch Neurol 67: 279-283, 2010.

4. Huang FY, Lee TW, Kao CH, et al: Imaging, autoradiography, and biodistribution of ${ }^{188} \mathrm{Re}$-labeled PEGylated nanoliposome in orthotopic glioma bearing rat model. Cancer Biother Radiopharm 26: 717-725, 2011

5. Miyata S, Kawabata S, Hiramatsu R, et al: Computed tomography imaging of transferrin targeting liposomes encapsulating both boron and iodine contrast agents by convection-enhanced delivery to F98 rat glioma for boron neutron capture therapy. Neurosurgery 68: 1380-1387, 2011.

6. Wolburg H, Wolburg-Buchholz K, Kraus J, et al: Localization of claudin-3 in tight junctions of the blood-brain barrier is selectively lost during experimental autoimmune encephalomyelitis and human glioblastoma multiforme. Acta Neuropathol 105 586-592, 2003.

7. Valk PE, Townsend DW and Maisey MN: Positron Emission Tomography: Basic Science and Clinical Practice. SpringerVerlag Publishing, New York, NY, 2003.

8. Strauss LG: Fluorine-18 deoxyglucose and false-positive results: a major problem in the diagnostics of oncological patients. Eur J Nucl Med 23: 1409-1415, 1996.

9. Kong XB, Zhu QY, Vidal PM, et al: Comparisons of anti-human immunodeficiency virus activities, cellular transport, and plasma and intracellular pharmacokinetics of 3'-fluoro-3'-deoxythymidine and 3'-azido-3'-deoxythymidine. Antimicrob Agents Chemother 36: 808-818, 1992.

10. Jensen MM, Erichsen KD, Johnbeck CB, et al: $\left[{ }^{18} \mathrm{~F}\right] \mathrm{FLT}$ and $\left[{ }^{18} \mathrm{~F}\right]$ FDG PET for non-invasive treatment monitoring of the nicotinamide phosphoribosyltransferase inhibitor APO866 in human xenografts. PLoS One 8: e53410, 2013.

11. Tjuvajev JG, Stockhammer G, Desai R, et al: Imaging the expression of transfected genes in vivo. Cancer Res 55: 6126-6132, 1995

12. Tjuvajev JG, Finn R, Watanabe K, et al: Noninvasive imaging of herpes virus thymidine kinase gene transfer and expression: a potential method for monitoring clinical gene therapy. Cancer Res 56: 4087-4095, 1996.

13. Borbath I, Gregoire V, Bergstrom M, Laryea D, Langstrom B and Pauwels S: Use of 5-[ $\left.{ }^{76} \mathrm{Br}\right]$ bromo-2'-fluoro-2'-deoxyuridine as a ligand for tumour proliferation: validation in an animal tumour model. Eur J Nucl Med Mol Imaging 29: 19-27, 2002.

14. Cho SY, Ravasi L, Szajek LP, et al: Evaluation of ${ }^{76} \mathrm{Br}-\mathrm{FBAU}$ as a PET reporter probe for HSV1-tk gene expression imaging using mouse models of human glioma. J Nucl Med 46: 1923-1930, 2005.

15. Kilbourn MR, Dence CS, Welch MJ and Mathias CJ: Fluorine-18 labeling of proteins. J Nucl Med 28: 462-470, 1987.
16. Gambhir SS, Barrio JR, Herschman HR and Phelps ME: Assays for noninvasive imaging of reporter gene expression. Nucl Med Biol 26: 481-490, 1999.

17. Jacobs AH, Li H, Winkeler A, et al: PET-based molecular imaging in neuroscience. Eur J Nucl Med Mol Imaging 30: 1051-1065, 2003.

18. Pan MH, Huang SC, Liao YP, et al: FLT-PET imaging of radiation responses in murine tumors. Mol Imaging Biol 10: 325-334, 2008

19. Wang HE, Liao AH, Deng WP, et al: Evaluation of 4-borono$2-{ }^{18} \mathrm{~F}$-fluoro-L-phenylalanine-fructose as a probe for boron neutron capture therapy in a glioma-bearing rat model. J Nucl Med 45: 302-308, 2004

20. Wang HE, Wu SY, Chang CW, et al: Evaluation of F-18-labeled amino acid derivatives and $\left.{ }^{18} \mathrm{~F}\right] \mathrm{FDG}$ as PET probes in a brain tumor-bearing animal model. Nucl Med Biol 32: 367-375, 2005.

21. Massoud TF and Gambhir SS: Molecular imaging in living subjects: seeing fundamental biological processes in a new light. Genes Dev 17: 545-580, 2003.

22. De A, Lewis XZ and Gambhir SS: Noninvasive imaging of lentiviral-mediated reporter gene expression in living mice. Mol Ther 7: 681-691, 2003.

23. Bryant MJ, Chuah TL, Luff J, Lavin MF and Walker DG: A novel rat model for glioblastoma multiforme using a bioluminescent F98 cell line. J Clin Neurosci 15: 545-551, 2008.

24. Chang YL, Wang HE, Liu RS, Pang F and Hwang JJ: Monitoring of tumor growth and metastasis potential in MDA-MB-435s/ tk-luc human breast cancer xenografts. Nucl Instrum Meth A 571: 155-159, 2007

25. Deng WP, Yang WK, Lai WF, et al: Non-invasive in vivo imaging with radiolabelled FIAU for monitoring cancer gene therapy using herpes simplex virus type 1 thymidine kinase and ganciclovir. Eur J Nucl Med Mol Imaging 31: 99-109, 2004.

26. Alauddin MM, Shahinian A, Park R, Tohme M, Fissekis JD and Conti PS: A general synthesis of 2'-deoxy-2'-[ $\left.{ }^{18} \mathrm{~F}\right]$ fluoro-5-methyl$1-\beta$-D-arabinofuranosyluracil and its 5-substituted nucleosides. J Labelled Compds Radiopharm 46: 285-289, 2003.

27. Kao CH, Xie HL, Liao CH, Chen WM and Kao PF: $\left[{ }^{18}\right.$ F]FBAU 3',5'-dibenzoate, a lipophilic prodrug, enhances brain uptake of the cell proliferation tracer $\left[{ }^{18} \mathrm{~F}\right] \mathrm{FBAU}$. Nucl Med Biol 35: 635-643, 2008.

28. Engelhorn T, Eyupoglu IY, Schwarz MA, et al: In vivo micro-CT imaging of rat brain glioma: a comparison with 3T MRI and histology. Neurosci Lett 458: 28-31, 2009.

29. Lin C, Itti E, Haioun C, et al: Early ${ }^{18} \mathrm{~F}-\mathrm{FDG}$ PET for prediction of prognosis in patients with diffuse large B-cell lymphoma: SUV-based assessment versus visual analysis. J Nucl Med 48: 1626-1632, 2007.

30. Tjuvajev JG, Avril N, Oku T, et al: Imaging herpes virus thymidine kinase gene transfer and expression by positron emission tomography. Cancer Res 58: 4333-4341, 1998.

31. Fu DX, Foss CA, Nimmagadda S, Ambinder RF and Pomper MG: Imaging virus-associated cancer. Curr Pharm Des 14: 3048-3065, 2008.

32. Miyagawa T, Gogiberidze G, Serganova I, et al: Imaging of HSV-tk reporter gene expression: comparison between $\left[{ }^{18} \mathrm{~F}\right]$ FEAU, $\left[{ }^{18} \mathrm{~F}\right] \mathrm{FFEAU}$, and other imaging probes. J Nucl Med 49: 637-648, 2008

33. Buursma AR, Rutgers V, Hospers GA, Mulder NH, Vaalburg W and de Vries EF: ${ }^{18}$ F-FEAU as a radiotracer for herpes simplex virus thymidine kinase gene expression: in-vitro comparison with other PET tracers. Nucl Med Commun 27: 25-30, 2006.

34. Rehemtulla A, Stegman LD, Cardozo SJ, et al: Rapid and quantitative assessment of cancer treatment response using in vivo bioluminescence imaging. Neoplasia 2: 491-495, 2000.

35. Jost SC, Collins L, Travers S, Piwnica-Worms D and Garbow JR: Measuring brain tumor growth: combined bioluminescence imaging-magnetic resonance imaging strategy. Mol Imaging 8: 245-253, 2009

36. Song HT, Jordan EK, Lewis BK, et al: Rat model of metastatic breast cancer monitored by MRI at 3 tesla and bioluminescence imaging with histological correlation. J Transl Med 7: 88, 2009.

37. Spaeth N, Wyss MT, Pahnke J, et al: Uptake of ${ }^{18} \mathrm{~F}$-fluorocholine, ${ }^{18} \mathrm{~F}$-fluoro-ethyl-L-tyrosine and ${ }^{18} \mathrm{~F}$-fluoro-2-deoxyglucose in F98 gliomas in the rat. Eur J Nucl Med Mol Imaging 33: 673-682, 2006. 